\title{
BEYOND THE STANDARD MODEL AT HERA: STATUS AND PROSPECTS
}

\author{
E. PEREZ * \\ CE-Saclay, DSM/DAPNIA/Spp, F-91191 Gif-sur-Yvette \\ and DESY, Notkestrasse 85, D-22607 Hamburg. \\ E-mail: eperez@hep.saclay.cea.fr
}

\begin{abstract}
An overview of experimental results on searches for new phenomena at HERA is presented. The complementarity with searches performed at other experiments is discussed and the prospects for a discovery, using the full HERA data to be delivered until mid-2007, are presented.
\end{abstract}

\section{Introduction}

Although remarkably confirmed so far by low and high energy experiments, the Standard Model (SM) of strong, weak and electromagnetic interactions remains unsatisfactory. Many models of "new physics" have been proposed to address the questions which are unexplained by the SM. Experimentally, the observation of deviations with respect to the SM predictions is a key part of the existing and future high energy programmes. At HERA, searches for new phenomena have been carried out using a luminosity of up to $280 \mathrm{pb}^{-1}$. Besides an excess of atypical $W$-like events observed at $\mathrm{H} 1$ all measurements are so far in good agreement with the SM expectation and constraints on models for new physics have been obtained.

\section{Searches for new phenomena in inclusive DIS}

Neutral Current Deep Inelastic Scattering (NC DIS) is measured at HERA for values of the photon virtuality $Q^{2}$ up to about $40000 \mathrm{GeV}^{2}$. Although the precision is still statistically limited at the highest $Q^{2}$, the good agreement of the measurements with the SM expectation allows stringent constraints on new physics to be set ${ }^{1}$. For example, a finite quark radius would reduce the high $Q^{2}$ DIS cross section with respect to the SM predictions, such that the current data rule out quark radii larger than $0.85 \cdot 10^{-18} \mathrm{~m}$, assuming that the electron is point-like. Similarly, the effective scale of

*Talk given at the Workshop "New Trends in HERA Physics", Ringberg (Germany), October 2-7 2005. 
eeqq contact interactions is constrained to be larger than typically $5 \mathrm{TeV}$ a similar sensitivity being achieved from the preliminary Run II Drell-Yan data of the Tevatron experiments ${ }^{2}$. The effective mass scale associated to the exchange of Kaluza-Klein gravitons in models assuming additional large space dimensions is constrained to be larger than about $0.8 \mathrm{TeV}$, slightly below the LEP and Tevatron bounds.

The longitudinal polarisation of the lepton beam in the HERA II data has been exploited to measure the polarisation dependence of the Charged Current (CC) DIS cross section ${ }^{3}$. Although these data constrain in principle the existence of a right-handed $W$ boson, the sensitivity is below the $W_{R}$ mass bounds obtained at the Tevatron. However these measurements confirm the left-handed nature of the weak interaction in the $t$-channel.

\section{Model-dependent searches}

As HERA is not an annihilation machine, the pair-production of new heavy particles, which could occur in $e^{+} e^{-}$or $p p$ collisions via their coupling to gauge bosons, has a very low cross-section at HERA. Instead, searches for the single production of new particles are performed at the H1 and ZEUS experiments. The cross-section for such processes depends on the unkown coupling of the new particle to the SM fields. Hence these searches do not provide absolute constraints on the mass of new particles. Conversely, the observation of a signal for the single production of a new particle would provide information not only on its mass, but also on this unknown coupling. In the following example constraints obtained on leptoquarks, squarks in $R$-parity violating supersymmetry and excited fermions are presented.

\subsection{Lepton-quark resonances: Leptoquarks}

An intriguing characteristic of the Standard Model is the observed symmetry between the lepton and the quark sectors, which is manifest in the representation of the fermion fields under the SM gauge groups, and in their replication over three family generations. This could be a possible indication of a new symmetry between the lepton and quark sectors, leading to "lepto-quark" interactions. Leptoquarks (LQs) are new scalar or vector colour-triplet bosons carrying both a baryon and a lepton number. Several types of LQs can be predicted, differing in their quantum numbers. The interaction of the LQ with a lepton-quark pair is parameterized by a coupling $\lambda$. Depending on its quantum numbers a LQ couples to $e q, \nu q$ or both. The branching ratio $\beta$ for a LQ to decay into an electron and a quark 


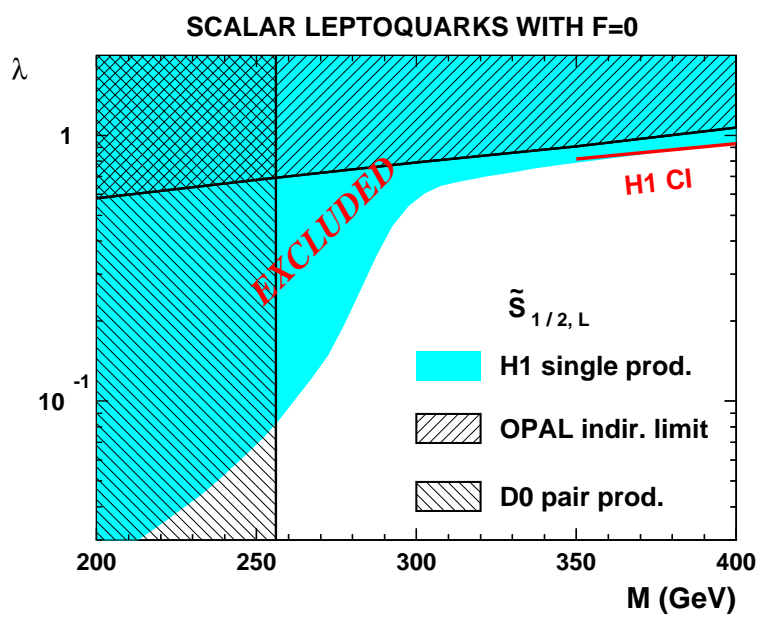

Figure 1. Example mass-dependent upper bounds on the Yukawa coupling $\lambda$ of a first generation scalar leptoquark to the electron-quark pair. These are shown for a LQ of $F=0$ coupling to an $e^{+}$and a $d$ quark, which decays exclusively into $e d$.

can be fixed by model assumptions, or can be treated as a free parameter. Decay modes other than $e q$ and $\nu q$ are usually neglected.

In electron-proton collisions, first generation LQs might be singly produced via the fusion of the incoming lepton with a quark coming from the proton. The production cross-section roughly scales with $\lambda^{2}$ times the parton density function of the relevant parton evaluated at $x_{B j}=M_{L Q}^{2} / s, \sqrt{s}$ being the centre-of-mass energy and $M_{L Q}$ the LQ mass. Hence, $e^{+} p\left(e^{-} p\right)$ collisions provide a larger sensitivity to LQs with fermion number $F=0$ $(F=2)$ since the $u$ and $d$ parton density is larger than that of antiquarks at large $x_{B j}$. LQs might be observed as a resonant peak in the lepton-jet mass spectrum of NC or CC DIS events. No such signal has been observed by the $\mathrm{H} 1$ and ZEUS experiments ${ }^{4}$.

Existing constraints on a scalar leptoquark which decays solely into an electron and a quark $(\beta=1)$ are summarised in Fig. 1. For an electromagnetic strength of the coupling $\lambda\left(\lambda^{2} / 4 \pi=\alpha_{e m}\right.$, i.e. $\left.\lambda \simeq 0.3\right)$, the HERA experiments rule out LQ masses below $\sim 290 \mathrm{GeV}$. Constraints derived from the search for pair produced LQs at the Tevatron do not depend on the coupling $\lambda$ and a lower mass bound of $256 \mathrm{GeV}$ is set by the D0 experiment ${ }^{5}$. Example constraints on more general LQ models where $\beta \neq 1$ are shown in Fig. 2 assuming that the LQ decays exclusively into 


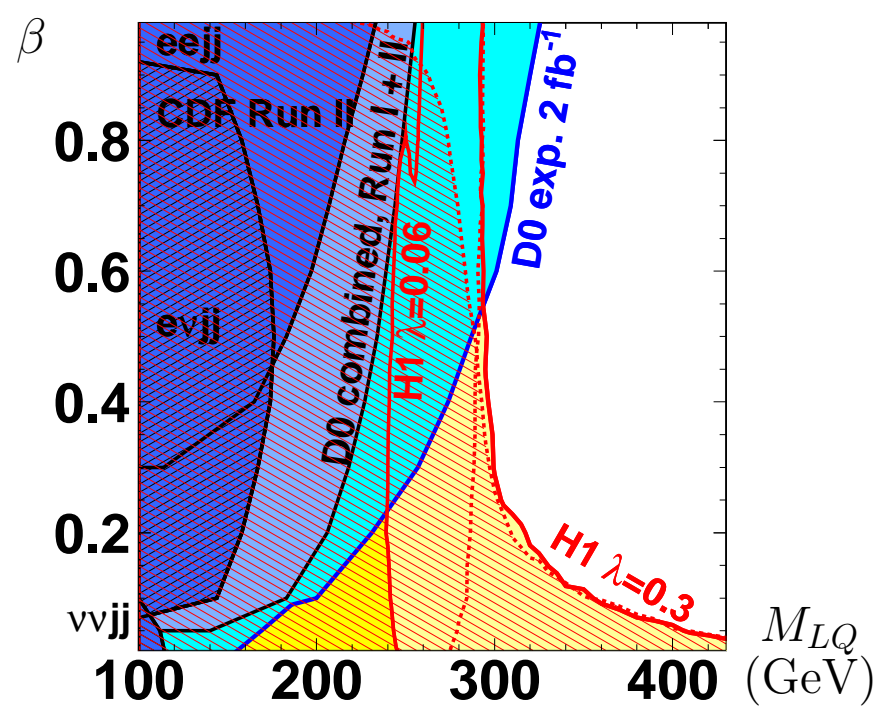

Figure 2. Example constraints on first generation scalar leptoquarks decaying exclusively into $e q$ and $\nu q$. For $\lambda=0.3$, the $\mathrm{H} 1$ constraints obtained from the $e j$ and $\nu j$ analyses are indicated by the dotted curves, and the combined bound is shown for two $\lambda$ values. The future sensitivity of the Tevatron for a luminosity of $2 \mathrm{fb}^{-1}$ is also shown.

$e q$ and $\nu q$. Leptoquarks decaying with a large branching ratio into $\nu q$ are not easily probed at the Tevatron due to the large background for final states containing only jets and missing transverse momentum. In contrast, with a similarly good signal to background ratio for the $e q$ and $\nu q$ final states, HERA experiments can be sensitive on LQs which decay with a large branching ratio into $\nu q$, provided that the coupling $\lambda$ is not too small.

The current HERA bounds are more stringent for $F=0$ LQs due to the much larger HERA I luminosity in $e^{+} p$ collisions (about $110 \mathrm{pb}^{-1}$ in $e^{+} p$ and $15 \mathrm{pb}^{-1}$ in $e^{-} p$ ). Assuming the existence of a $F=0$ LQ in the HERA kinematic range, and that its coupling to the $e q$ pair is equal to the HERA I upper limit, a significance of $\sim 4 \sigma$ could be reached in each experiment with an $e^{+} p$ luminosity of $350 \mathrm{pb}^{-1}$ and combining the $\mathrm{H} 1$ and ZEUS data could allow the $5 \sigma$ threshold to be reached. Assuming a LQ production crosssection equal to half the existing limit the discovery potential is limited. In contrast a much larger discovery potential remains for $F=2$ leptoquarks, which will be probed with the $e^{-} p$ data to be delivered until mid-2007.

It should be noted that an $e p$ collider would be the ideal machine to study a leptoquark signal. The fermion number of the leptoquark could 
easily be determined by comparing the signal rates in $e^{+} p$ and $e^{-} p$ collisions; the polarisation of the lepton beam provides a handle to determine the chiral couplings of the LQ; and the good signal to background ratio in the $\nu q$ channel allows the LQ coupling to the neutrino to be studied.

\subsection{R-parity violating supersymmetry}

In supersymmetric (SUSY) models where the so-called $R$-parity $\left(R_{p}\right)$ is not conserved, squarks could be resonantly produced at HERA similarly to leptoquarks. In addition to the "LQ-like" decays into $e q$ and possibly $\nu q$ the squarks also undergo decays into gauginos (the supersymmetric partners of gauge bosons) and an exhaustive search requires a large number of final states to be analysed. This has been pioneered by the H1 collaboration in ${ }^{6}$ where the full HERA I dataset has been used to set constraints on supersymmetric models. A similar preliminary analysis looking for the SUSY partner of the top quark has been performed by the ZEUS experiment. Example constraints are shown in Fig. 3. For a squark $R_{p}$-violating coupling

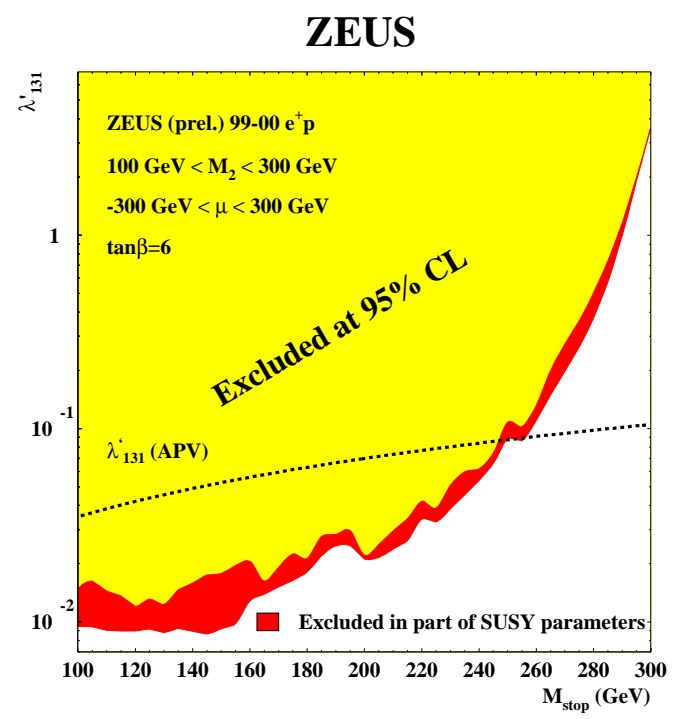

Figure 3. Example mass-dependent constraints on the coupling of the stop to an $e^{+} d$ pair. A scan of the SUSY parameter space has been performed. The light shaded domain is ruled out at $95 \%$ confidence level for any value of the parameters, which determine the dominant final states in which the signal could be observed. As can be seen from the narrowness of the dark band, the sensitivity of the analysis is nearly model-independent. 
of the electromagnetic strength, lower mass bounds of $270-280 \mathrm{GeV}$ can be set. Within constrained SUSY models where a few parameters determine the full Higgs and supersymmetric spectrum, stringent bounds were derived on the squark mass from searches for Higgs, sfermions and gauginos at LEP. However, part of the SUSY parameter space remains open for a discovery at HERA II, for a reasonably large $R_{p}$ coupling ${ }^{6}$. The case of a light stop or sbottom is of high interest for HERA II since the bounds coming from Tevatron are less stringent than those obtained assuming five degenerate squarks. In particular, the sensitivity on the sbottom, which has a larger production cross-section in $e^{-} p$ than in $e^{+} p$ collisions $\left(e^{-} u \rightarrow \tilde{b}\right)$, will considerably increase with the HERA II $e^{-} p$ data.

In case the squarks are too heavy to be produced at HERA, the $t$ channel exchange of a selectron or sneutrino could allow for single gaugino production. This process has been considered in two classes of SUSY models, differing in the dominant decay mode of the produced gaugino ${ }^{7}$. In both cases the analyses slightly improve the previous bounds if the relevant R-parity violating coupling is quite large. These are the first SUSY constraints set at HERA which are independent of the squark sector.

\subsection{Fermion-boson resonances}

The observed replication of three fermion families motivates the possibility of a yet unobserved new scale of matter. An unambiguous signature for a new scale of matter would be the direct observation of excited states of fermions $\left(f^{*}\right)$, via their decay into a fermion and a gauge boson. In the most commonly used model ${ }^{8}$, the interaction of an $f^{*}$ with a fermion and a gauge boson is described by a magnetic coupling proportional to $1 / \Lambda$ where $\Lambda$ is a new scale. Proportionality constants $f, f^{\prime}$ and $f_{s}$ result in different couplings to $U(1), S U(2)$ and $S U(3)$ gauge bosons. Existing constraints on excited electrons are shown in Fig. 4, under the assumption that $f=f^{\prime}$. Searches for pair produced $e^{*}$ at LEP allowed to rule out masses below about $103 \mathrm{GeV}$, independently of the value of the coupling $f / \Lambda$. In contrast, searches for single $e^{*}$ production at LEP, HERA ${ }^{9}$ and Tevatron ${ }^{10}$ set mass bounds which depend on $f / \Lambda$. The future HERA and Tevatron sensitivities, also depicted in Fig. 4, show the discovery potential of HERA II for excited electrons. The case of excited neutrinos is also very interesting for HERA II, since their production cross-section is larger in $e^{-} p$ than in $e^{+} p$ collisions by typically one order of magnitude. 


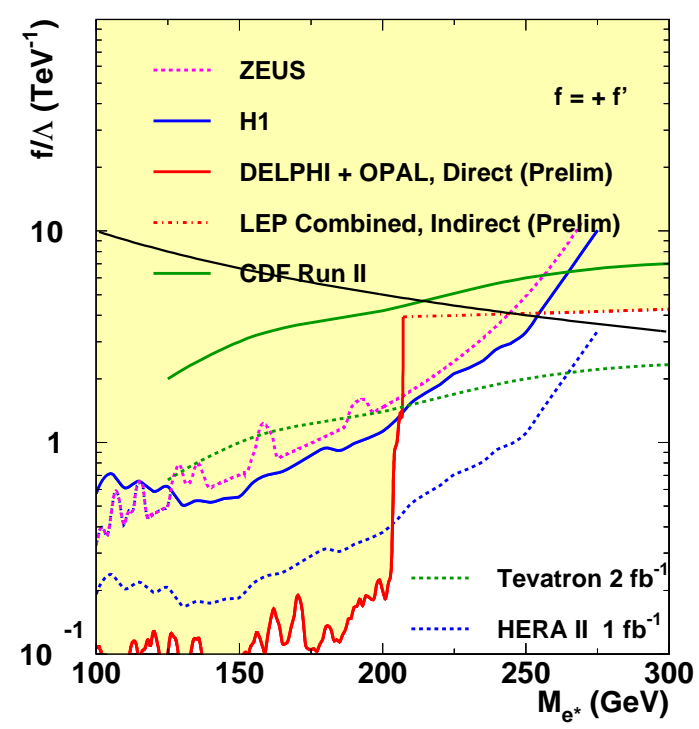

Figure 4. Existing constraints on excited electron masses and couplings, assuming that $f=f^{\prime}$. The decreasing curve shows the hyperbola $f / \Lambda=1 / M_{e^{*}}$. The future HERA and Tevatron sensitivities are also shown as dotted curves.

\section{Searches for deviations from the $\mathrm{SM}$ in rare processes}

\subsection{The "isolated lepton events"}

Within the Standard Model, $W$ production at HERA has a cross-section of about $1 \mathrm{pb}$. When the $W$ decays leptonically, the final state contains an isolated lepton, missing transverse momentum, and a usually soft hadronic system. This process has been measured using the HERA I data ${ }^{11}$ in the "electron" $\left(W \rightarrow e \nu_{e}\right)$ and "muon" $\left(W \rightarrow \mu \nu_{\mu}\right)$ channels, and a general agreement with the SM prediction was observed. However, for large values of the transverse momentum of the hadronic system, $P_{T}^{X}$, an excess of events was reported by the H1 Collaboration ${ }^{11}$. This excess was not confirmed by a ZEUS analysis ${ }^{12}$, differing from the $\mathrm{H} 1$ analysis in terms of background rejection ${ }^{\mathrm{a}}$.

An abnormally large rate of high $P_{T}^{X}$ events is also observed by the H1 experiment ${ }^{13}$ in the HERA II data. Combining the $e$ and $\mu$ channels and the HERA I and HERA II datasets, which amount to a total luminosity

a The non $W$ contribution to the expected background was about $50 \%$ at large $P_{T}^{X}$ in the ZEUS analysis, while it amounts to $15 \%$ only in the $\mathrm{H} 1$ analysis. 

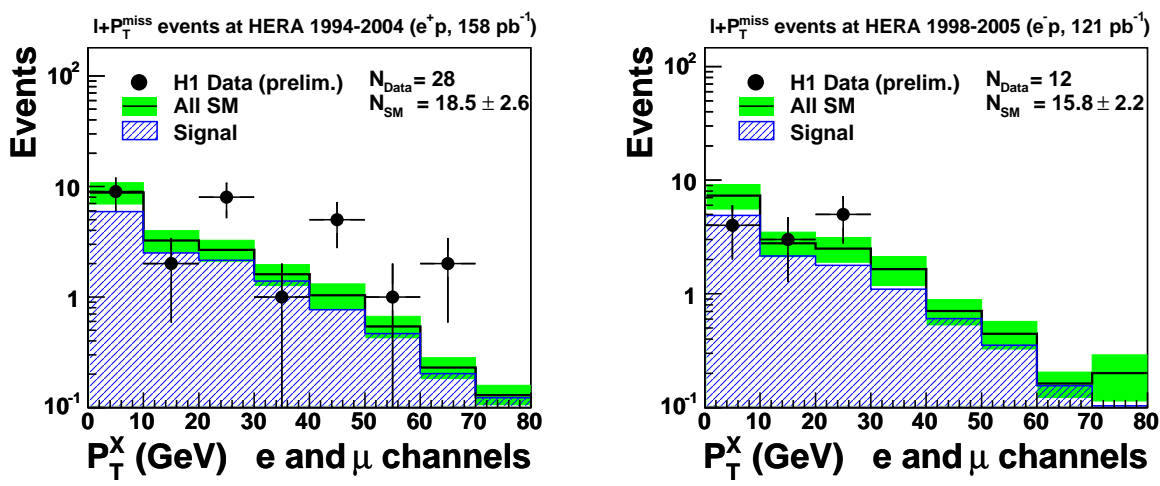

Figure 5. Distribution of the transverse momentum of the hadronic system $P_{T}^{X}$ in selected events recorded in (left) the $e^{+} p$ data sample and (right) the $e^{-} p$ data sample. The hatched histogram shows the expectation from $W$ production while the total expectation is given by the open histogram.

of $279 \mathrm{pb}^{-1}, 17$ events are observed at $P_{T}^{X}>25 \mathrm{GeV}$ for a SM expectation of $9.0 \pm 1.5$. Amongst the 6 new events observed in HERA II, 5 were recorded during the $e^{+} p$ running $\left(53 \mathrm{pb}^{-1}\right)$ and one during the $e^{-} p$ running $\left(107 \mathrm{pb}^{-1}\right)$. Fig. 5 shows the observed $P_{T}^{X}$ distributions separately for the $e^{+} p$ and $e^{-} p$ datasets where HERA I and HERA II data are combined, together with the corresponding SM expectations. The observed and expected numbers of events are given in table 1 . While the observation in the $e^{-} p$ data is consistent with the SM expectation, 15 events are observed at $P_{T}^{X}>25 \mathrm{GeV}$ in the $e^{+} p$ data for an expectation of $4.6 \pm 0.8$ events. The probability that the expected yield fluctuates to 15 events or more corresponds to a $3.4 \sigma$ fluctuation.

The ZEUS experiment has recently carried out a re-analysis of the $e$ channel using the 99-00 $e^{+} p$ data, resulting in a larger purity in $W$ events. The positron data taken at HERA II have been analysed in the same way, such that the total luminosity amounts to $106 \mathrm{pb}^{-1}$. The results are also shown in table 1. At $P_{T}^{X}>25 \mathrm{GeV}$ one event is observed in the data, in agreement with the SM expectation of $1.5 \pm 0.18$.

Although the rate of events observed in the $e$ channel in the positron data is larger in H1 than in ZEUS, both experiments are compatible with each other within $2.5 \sigma$ for an average rate of about 4 events per $100 \mathrm{pb}^{-1}$ in this channel. Assuming that such events are observed in the future H1 and ZEUS data at an average rate of about $7-8$ events per $100 \mathrm{pb}^{-1}$ 
combining the $e$ and $\mu$ channels, a significance of $4 \sigma$ could be reached $^{\mathrm{b}}$ from the combined H1 and ZEUS datasets by doubling the $e^{+} p$ luminosity. It should be noted that new physics scenarios can be found which could explain that such events are observed in $e^{+} p$ collisions only. For example, in supersymmetry with two $R$-parity violating couplings involving third generation fields, a top quark could be produced via $t$-channel sbottom exchange in $e^{+} d$ collisions. Due to the large value of Bjorken $x$ needed to produce a top quark in the final state, the corresponding process in $e^{-} p$ collisions would have a much lower cross-section.

Table 1. Summary of the H1 results of searches for events with isolated electrons or muons and missing transverse momentum for the $e^{+} p$ data $\left(158 \mathrm{pb}^{-1}\right)$ and the $e^{-} p$ data $(121$ $\left.\mathrm{pb}^{-1}\right)$. Data from HERA I and HERA II are combined. The number of events observed by ZEUS in the electron channel, in $106 \mathrm{pb}^{-1}$ of $e^{+} p$ data, is also shown. The number of observed events at $P_{T}^{X}>25 \mathrm{GeV}$ is compared to the $\mathrm{SM}$ prediction. The quoted errors contain statistical and systematic uncertainties added in quadrature.

\begin{tabular}{|c|c|c|c|c|}
\hline \multicolumn{1}{|c|}{$P_{T}^{X}>25 \mathrm{GeV}$} & $\begin{array}{c}\text { Electron } \\
\text { obs./exp. }\end{array}$ & $\begin{array}{c}\text { Muon } \\
\text { obs./exp. }\end{array}$ & $\begin{array}{c}\text { Combined } \\
\text { obs./exp. }\end{array}$ \\
\hline \hline $\begin{array}{c}\text { H1 } \\
\text { Preliminary }\end{array}$ & $\begin{array}{c}1998-2005 e^{-} p \\
121 \mathrm{pb}^{-1}\end{array}$ & $2 / 2.4 \pm 0.5$ & $0 / 2.0 \pm 0.3$ & $2 / 4.4 \pm 0.7$ \\
\hline \hline $\begin{array}{c}\text { H1 } \\
\text { Preliminary }\end{array}$ & $\begin{array}{c}1994-2004 e^{+} p \\
158 \mathrm{pb}^{-1}\end{array}$ & $9 / 2.3 \pm 0.4$ & $6 / 2.3 \pm 0.4$ & $15 / 4.6 \pm 0.8$ \\
\hline \hline $\begin{array}{c}\text { ZEUS } \\
\text { Preliminary }\end{array}$ & $\begin{array}{c}1999-2004 e^{+} p \\
106 \mathrm{pb}^{-1}\end{array}$ & $1 / 1.5 \pm 0.18$ & \multicolumn{2}{|c}{} \\
\cline { 2 - 3 }
\end{tabular}

\subsection{Multi-lepton events}

If the events reported above were to be explained by some anomalous $W$ production mechanism, an anomalous rate for $Z$-like events could also be observed. Events with at least two electrons or muons in the final state have been looked for by the $\mathrm{H} 1$ collaboration ${ }^{14}$. A slight excess of high mass multi-electron events was observed in the HERA I dataset. The analysis has been repeated using the HERA II data and extended to include other multi-lepton topologies ${ }^{15}$. With a total luminosity of $209 \mathrm{pb}^{-1}$ four events are observed with $\sum_{\text {leptons }} P_{T}>100 \mathrm{GeV}$, three of which being HERA I $e e$ events. This is slightly above the SM prediction of $0.81 \pm 0.14$.

${ }^{\mathrm{b}}$ This estimate is obtained by scaling the SM backgrounds of the H1 analysis in both the $e$ and $\mu$ channels. 


\section{Conclusions}

Although some stringent bounds on new physics are set at LEP and the Tevatron, HERA appears to be very well suited to search for new phenomena in some specific cases. In particular, searches for new physics at HERA rarely suffer from huge SM backgrounds. Searches for leptoquarks, for the supersymmetric partners of the top or bottom quarks, and for excited fermions might bring a discovery with the HERA II data. This holds in particular for new physics processes for which the cross-section is larger in $e^{-} p$ than in $e^{+} p$ collisions, since the HERA I constraints are not too stringent in such cases. With the future $e^{+} p$ data to be delivered until mid-2007, the excess of atypical $W$-like events observed at H1, which corresponds to a $3.4 \sigma$ deviation, appears to be the best chance for a discovery at HERA II.

\section{References}

1. H1 Collab., C. Adloff et al., Phys. Lett. B568 (2003) 35; ZEUS Collab., S. Chekanov et al., Phys. Lett. B591 (2004) 23.

2. D0 Collab., D0 Note 4552-CONF.

3. J. Meyer, these proceedings.

4. H1 Collab., Phys. Lett. B629 (2005) 9; ZEUS Collab., S. Chekanov et al., Phys. Rev. D68 (2003) 052004.

5. D0 Collab., V.M. Abazov et al., Phys. Rev. D71 (2005) 071104.

6. H1 Collab., A. Aktas et al., Eur. Phys. J. C36 (2004) 425.

7. H1 Collab., A. Aktas et al., Phys. Lett. B616 (2005) 31; ZEUS Collab., contributed paper to EPS'05, abstract \#329; idem, contributed paper to EPS'05, abstract \#330.

8. K. Hagiwara, D. Zeppenfeld and S. Komamiya, Zeit. fur Phys. C29 (1985) 115; F. Boudjema, A. Djouadi and J.L. Kneur, Zeit. fur Phys. C57 (1993) 425.

9. H1 Collab., C. Adloff et al., Phys. Lett. B548 (2002) 35; ZEUS Collab., S. Chekanov et al., Phys. Lett. B549 (2002) 32.

10. CDF Collab., D. Acosta et al., Phys. Rev. Lett. 94 (2005) 101802.

11. H1 Collab., V. Andreev et al., Phys. Lett. B561 (2003) 241.

12. ZEUS Collab., S. Chekanov et al., Phys. Lett. B559 (2003) 153.

13. H1 Collab., document prepared for the Nov. 2005 DESY-PRC meeting, available at http://www-h1.desy.de/publications/H1preliminary.short_list.html.

14. H1 Collab., A. Aktas et al., Eur. Phys. J. C31 (2003) 17; H1 Collab., A. Aktas et al., Phys. Lett. B583 (2004) 28.

15. H1 Collab., contributed to EPS'05, abstract \#635. 